Bulletin of the Section of Logic

Volume 45:3/4 (2016), pp. 257-267

http://dx.doi.org/10.18778/0138-0680.45.3.4.08

Wojciech Dzik and Sándor Radeleczki

\title{
PRESERVING FILTERING UNIFICATION BY ADDING COMPATIBLE OPERATIONS TO SOME HEYTING ALGEBRAS
}

\begin{abstract}
We show that adding compatible operations to Heyting algebras and to commutative residuated lattices, both satisfying the Stone law $\neg x \vee \neg \neg x=1$, preserves filtering (or directed) unification, that is, the property that for every two unifiers there is a unifier more general then both of them. Contrary to that, often adding new operations to algebras results in changing the unification type. To prove the results we apply the theorems of [9] on direct products of $l$-algebras and filtering unification. We consider examples of frontal Heyting algebras, in particular Heyting algebras with the successor, $\gamma$ and $G$ operations as well as expansions of some commutative integral residuated lattices with successor operations.

Keywords and phrases: filtering unification, compatible operation, intuitionistic logic, Heyting algebra, residuated lattice

\section{Introduction}

Unification in general, and unification types, in particular, very much depend on the signature, the set of basic operations or connectives. Generally speaking, unification of terms is concerned with finding a substitution for variables such that, after substituting, the equality of the terms become provable in a given (equational) theory. We recall that a substitution $\sigma$ of a finite number of variables is a unifier for terms $t_{1}$ and $t_{2}$ in a theory $T$ if $\vdash_{T} \sigma\left(t_{1}\right)=\sigma\left(t_{2}\right)$. In this case $t_{1}$ and $t_{2}$ are unifiable. A substitution $\sigma_{1}$ is more general than a substitution $\sigma_{2}$ if there exists a substitution $\tau$
\end{abstract}


such that $\vdash_{T} \tau\left(\sigma_{1}(x)\right)=\sigma_{2}(x)$, for any $x$ from a finite set of variables. A most general unifier (a $m g u$ ) for terms $t_{1}, t_{2}$ is a unifier for $t_{1}, t_{2}$ which is more general than any unifier for $t_{1}, t_{2}$. Unification in $T$ is unitary if any unifiable terms have a mgu. The other extreme is the case in which there exist unifiable terms $t_{1}, t_{2}$ such that a maximal unifier for them does not exist, in this case unification is nullary. Finitary unification means that there are finitely many maximal unifiers for every unifiable terms. There is the fourth, infinitary type, which will not appear in this paper. For more details and complete definitions see [2] and [1]. Since many attempts to characterize the unitary unification type failed, Ghilardi and Sacchetti [14] introduced filtering unification, which is a property that for every two unifiers there is a unifier more general then both of them. They managed to characterize modal logics with filtering unification, see [14].

To see that changes of the signature can change (even filtering) unification let us consider some reducts of intuitionistic logic, for example. Unification in the following fragments of intuitionistic logic: $\rightarrow$-fragment, $\rightarrow, \wedge$-fragment, $\rightarrow, \wedge, \neg$-fragment is unitary (hence, filtering), but in $\rightarrow, \neg$ fragment it is not unitary, see [18], but finitary, see [7] (hence not filtering). Moreover in full language with $\rightarrow, \wedge, \vee, \neg$ unification is not unitary but finitary, see [13] (not filtering). By removing $\rightarrow$ and $\neg$ we get distributive lattices with nullary unification. For another example take Boolean algebras, in which unification is unitary (i.e. filtering) but adding a topological interior operation, which corresponds to the S4-modal connective of necessity, leads to interior algebras that have finitary unification (not filtering) see [15]. Hence adding or removing one or more connectives changes (even filtering) unification. There are many examples of this kind.

The problem of adding new connectives in a natural way, especially to intuitionistic logic, has been studied by several authors, see e.g. [10], [4] for references. From the point of view of algebraic semantics it is natural to demand that the new connective considered as an operation on Heyting algebras should preserve congruences of the algebras, that is, the connective is compatible. An operation $f$ is called compatible in an algebra $\mathcal{A}$ if it is compatible with (or preserves) every congruence of $\mathcal{A}$, that is, if every congruence of $\mathcal{A}$ is a congruence of $(\mathcal{A} ; f)$. In particular, every polynomial is compatible. Are there any compatible operations which are different from polynomials? In Boolaen algebras all compatible operations are polynomials, this is why Boolaen algebras are called affine complete. Heyting algebras are not affine complete, there are many interesting addi- 
tional compatible connectives, like the frontal operators, the successor, $\gamma$ and $G$ operations, studied in many papers, see [10] and [4] and [6] for more references.

In [8] it is shown that a variety of Heyting algebras have filtering unification iff it satisfies the Stone identity $\neg x \vee \neg \neg x=1$ (hence unification is unitary or nullary). In this case one can not determine whether unification remains filtering after adding some extra operations to algebras or after adding new connectives to an intermediate logic. Similarly, in [9] it is shown that varieties of commutative integral residuated lattices which satisfy the Stone identity $\neg x \vee \neg \neg x=1$ have filtering unification, hence unification is unitary or nullary.

This paper is based on an observation that the results of [9] can be applied to show that unification remains filtering in any new variety of Heyting algebras or of commutative integral residuated lattices (both satisfying the Stone identity) which results by adding extra compatible operations.

Below we include, as examples, several widely investigated varieties of Heyting algebras (and some commutative integral residuated lattices) with additional compatible operations, for which filtering unification is preserved. To support the claim that adding compatible operations (or connectives) makes significant changes in unification, we provide formulas (terms) which are consistent but not unifiable. In any intermediate logic (without additional connectives) unifiable formulas and consistent formulas coincide.

Up to our knowledge unification in varieties with additional compatible operations has not been investigated yet.

\section{Preliminaries}

We recall some basic notions from e.g. [9]. Since almost all are standard they can be also found in other sources, see e.g. [3].

Let $L$ be a bounded lattice with 0 and 1 . An element $a \in L$ is called a central element of $L$ if $a$ is complemented and for all $x, y \in L$ the sublattice generated by $\{a, x, y\}$ is distributive. We denote by $\operatorname{Cen}(L)$ the set of all central elements in $L$, a Boolean sublattice of the lattice $L$. Each $c \in \operatorname{Cen}(L)$ has a single complement denoted by $\bar{c} \in \operatorname{Cen}(L)$; the pair $\{c, \bar{c}\}$ is called a central pair of $L$. Each $c \in \operatorname{Cen}(L)$, induces a congruence $\theta_{c}=\{(x, y) \mid x \wedge c=y \wedge c\}$, for any $c \in \operatorname{Cen}(L)$, and $\theta_{c}$ and $\theta_{\bar{c}}$ form a pair of factor congruences of $L$ (in [3]: congruences $\theta, \theta^{*}$ form a pair of factor 
congruences if they permute, $\theta \cap \theta^{*}=\triangle$ and $\theta \vee \theta^{*}=\nabla$ ); and if $\theta_{1}$ and $\theta_{2}$ are factor congruences of a bounded lattice $L \cong L / \theta_{1} \times L / \theta_{2}$, then $\theta_{1}=\theta_{c}$, $\theta_{2}=\theta_{\bar{c}}$, for some $c \in \operatorname{Cen}(L)$.

An algebra $\mathcal{A}=(L, \wedge, \vee, 0,1, F)$ is called an l-algebra if $(L, \wedge, \vee, 0,1)$ is a bounded lattice, and any $n$-ary term $f: L^{n} \rightarrow L$ of $\mathcal{A}$ is centre-preserving, that is, for every $c \in \operatorname{Cen}(L)$,

$$
\left(x_{i}, y_{i}\right) \in \theta_{c}, 1 \leq i \leq n \text { implies }\left(f\left(x_{1}, \ldots, x_{n}\right), f\left(y_{1}, \ldots, y_{n}\right)\right) \in \theta_{c} .
$$

A congruence $\theta$ of $\operatorname{Con} \mathcal{A}$ is said to be compact if for every $(\emptyset \neq) \Phi \subseteq$ $\operatorname{Con} \mathcal{A}, \theta \leq \bigvee \Phi \Rightarrow \theta \leq \bigvee F$, for some finite $F \subseteq \Phi$.

An algebra $\mathcal{A}=(A, F)$ with a constant 1 is called 1-regular (or weakly regular), if for each $\varphi, \theta \in \operatorname{Con} \mathcal{A}:[1]_{\varphi}=[1]_{\theta} \Rightarrow \varphi=\theta$.

Examples of $l$-algebras include bounded lattices, p-algebras, ortholattices, Heyting algebras etc., and also: residuated lattices (as shown in [9] ). Note that modal (e.g. S4) algebras are not, in general, l-algebras (hence the result in [9] does not generalize the result of [14]).

Any $l$-algebra is congruence distributive and the factor congruences of an $l$-algebra and of its underlying lattice $L$ coincide.

In the following $\mathcal{V}$ is a 1 -regular variety of $l$-algebras of the form $\mathcal{A}=$ $(L, \wedge, \vee, 0,1, F)$ satisfying the following properties:

(A) Each $(L, \wedge, \vee, 0,1)$ is a bounded lattice such that any $u, v \in L$ which are the complements of each other in $L$ are central elements in $L$.

(B) Each algebra $\mathcal{A} \in \mathcal{V}$ has a unary term $g$ such that for any $v \in L$ $v \wedge g(v)=0$ and $g(0)=1$,

(C) Each algebra $\mathcal{A}=(L, \wedge, \vee, 0,1, F) \in \mathcal{V}$ has two unary terms $h$ and \rceil $h$, such that for every $v \in L, h(v)$ and $\rceil h(v)$ are the complements of each other in the lattice $L$, and $h(0)=\urcorner h(1)=1$.

It is easy to see that condition (A) is satisfied in any bounded distributive lattice. Moreover, in [9; Theorem 15] we proved that every bounded commutative residuated lattices satisfies (A). Therefore, any Heyting algebra is an $\ell$-algebra satisfying condition (A).

The main result of the paper [9] is the following

TheOREM 1. Let $\mathcal{V}$ be a variety of 1-regular l-algebras. If the conditions $(A),(B)$ and $(C)$ are satisfied by the algebras of $\mathcal{V}$, then unification in $\mathcal{V}$ is filtering. 
One of the applications of the above theorem in [9] is

THEOREM 2. Unification in commutative integral (even non-distributive) residuated lattices satisfying the Stone identity $\neg \neg x \vee \neg x=1$ is filtering, that is, unitary or nullary.

For an algebra $H$, an operation $f: H^{n} \rightarrow H$ is compatible with $H$ if it is compatible with every congruence $\theta$ of $H$, that is: if $x_{i} \theta y_{i}$, for $i \leq n$, then $f\left(x_{1}, \ldots, x_{n}\right) \theta f\left(y_{1}, \ldots, y_{n}\right)$.

The conditions on $l$-algebras of the form $\mathcal{A}=(L, \wedge, \vee, 0,1, F)$ require that operations in $F$ should be centre-preserving. Thus compatible operations, which preserve all congruences of $L$, satisfy these conditions. Assume now that $\mathcal{A}$ satisfies conditions (B) and (C). Observe that, after adding new compatible operations to $F$, these conditions still remain valid for the obtained algebra. Hence we can extend the result by allowing expansions of Heyting algebras and of commutative integral (even non-distributive) residuated lattices with compatible operations.

In [7] it was proved that unification in Heyting algebras satisfying the Stone identity is filtering (see also [9]). Now, since any bounded distributive lattice satisfies condition (A), this result can be reformulated as follows:

COROLLARY 3. Unification in a variety of Heyting algebras satisfying the Stone identity $\neg \neg x \vee \neg x=1$ and expanded with compatible operations is filtering, and hence, it is unitary or nullary.

Similarly, by checking assumptions (A), (B) and (C) of Theorem 1 for commutative integral residuated lattices (the definition is postponed till the next section) we conclude that

THEOREM 4. Unification in commutative integral (even non-distributive) residuated lattices satisfying the Stone identity $\neg \neg x \vee \neg x=1$ and expanded with compatible operations is filtering, that is, unitary or nullary.

\section{Heyting algebras with compatible operations: frontal, the sucessor, $\gamma$ and $G$. Bounded integral commutative residuated lattices with compatible operations}

In intuitionistic propositional logic INT, none of the basic connectives $\{\wedge, \vee, \rightarrow, \neg\}$ (or $\{\wedge, \vee, \rightarrow, \perp\}$ ) is definable by the other, the result of 
M. Wajsberg of 1938. There are several connectives that are compatible and also new, as they can not be defined in terms of the basic connectives.

Expansions of Heyting algebras (equivalently expansions of INT), with compatible operations (or connectives) were investigated by many authors, see e.g. [10], [4], [6] for more references. Now we recall some important examples to show also that they have various motivations: topological, provability logic, successor, dense elements etc.

Frontal Heyting algebras. L. Esakia [10] considered the modal Heyting calculus $m H C$. The corresponding variety of algebras is the class of frontal Heyting algebras that is algebras of the form $(H, \wedge, \vee, \rightarrow, \tau, 0,1)$, where $(H, \wedge, \vee, \rightarrow, 0,1)$ is a Heyting algebra and $\tau$ is a unary operation, called frontal, which satisfies the following equations:

(f1) $\tau(x \wedge y)=\tau(x) \wedge \tau(y)$,

(f2) $x \leq \tau(x)$,

(f3) $\tau(x) \leq y \vee(y \rightarrow x)$.

In a topological semantics $\tau$ is interpreted as the dual to the CantorBendixson derivative in scattered spaces, see [10]. Let us observe that every frontal operation $\tau$ is a compatible operation since, by (f1) and (f2), $\tau$ satisfies $\tau(x \wedge y) \wedge y=\tau(x) \wedge y$, which is the condition for $\tau$ to be the compatible operation in a Heyting algebra, see [4].

Now we present three important examples of frontal operations.

The Successor operation S. Let $H$ be a Heyting algebra and assume that an operation $S$ satisfies the following equations:

(S1) $x \leq S(x)$,

(S2) $S(x) \leq y \vee(y \rightarrow x)$,

(S3) $S(x) \rightarrow x=x$.

$S(x)$ is called then the successor of $x$ in $H$. Since (S1) and (S3) are equivalent to (S4): $S(x) \rightarrow x \leq x$, one can observe that the successor $S$ is a frontal operation satisfying (S4), see [6]. $S$ can be also given by the following definition: $S(x)=\min \{y: y \rightarrow x \leq x\}$. The corresponding axioms for the logic of the successor $S$ are:

S'1. $A \rightarrow S A$

S'2. $S A \rightarrow(B \vee(B \rightarrow A))$

S'3. $(S A \rightarrow A) \rightarrow A$ 
For a finite chain $H_{n}=\{0,1, \ldots, k\}$ of $(k+1)$ elements treated as a Heyting algebra with the natural order, i.e. $\perp=0, \top=n, \wedge=\min , \vee=\max$, $a \rightarrow b=b$ if $a>b$, or $=1$ otherwise, we have: $S(k)=k+1$, for $k \neq n$ and $S(\top)=S(n+1)=n+1=\top$. The operation $S$ mimics in Heyting algebras (especially chains) the successor known from natural numbers. The connective $S$ was introduced earlier by Kuznetsov in 1985, as an irreflexive modality $\square$ satisfying the axioms S'1 - S'3 (where $S$ is replaced by $\square$ ) with the aim of investigating an intuitionistic version of the provability logic GL, see [17]. Unification in "classical" version of GL is finitary, see [15].

The operation $\gamma$. The operation $\gamma$ was introduced in [4] as a compatible operation in Heyting algebras with the meaning: $\gamma(x)$ is the smallest dense element above an element $x$ in $H$.

Again, $\gamma$ can be also defined as a frontal operation satisfying additionally $(\mathrm{C} 1)$ and also additionally $(\mathrm{C} 4): \gamma(x) \leq x \vee \gamma(0)$, see [6]. The corresponding axioms in logic are:

C'1. $\neg \neg \gamma A$

C'2. $A \rightarrow \gamma A$

C'3. $\gamma A \rightarrow(A \vee B \vee \neg B)$

C'4. $(A \rightarrow B) \rightarrow(\gamma A \rightarrow \gamma B)$.

$\gamma(x)$ can be also introduced by the definition: $\gamma(x)=\min \{y: \neg y \vee x \leq y\}$.

The operation $G$. The operation $G$ was introduced by D. Gabbay as a compatible connective. In $[6]$ it is characterized by the following equations:

(G1) $(G(x) \rightarrow x) \vee \neg \neg x \leq G(x)$,

(G2) $G(x) \leq y \vee((y \rightarrow x) \wedge \neg \neg x)$,

The corresponding axioms in logic are:

G1. $G A \rightarrow(B \vee(B \rightarrow A))$

G2. $(A \rightarrow B) \rightarrow(G A \rightarrow G B)$

G3. $(A \rightarrow G A)$

G4. $(G A \rightarrow \neg \neg A)$

G5. $(G A \rightarrow A) \rightarrow(\neg \neg A \rightarrow A)$.

The Gabbay's operation $G$ can be also characterized as a frontal operation satisfying the equation $G(x) \rightarrow x=\neg \neg x$, see [6].

It is known, see [4], that $\gamma$ and $G$ are definable in terms of $S$, but $S$ is not definable in terms of $\gamma$ and $G$. The operations $S, \gamma$ and $G$ do not exist in the Heyting algebra based on the unit interval $[0,1]$, hence they 
are not definable, they are not Heyting polynomials, so they are new. For more about compatible operations see [4] and [6].

Theorem 5. Let $\mathcal{V}^{*}$ be an expansion of algebras of any variety $\mathcal{V}$ of Heyting algebras with new compatible operations. If $\neg \neg x \vee \neg x=1$ holds in $\mathcal{V}$, then unification in $\mathcal{V}^{*}$ is filtering, hence it is unitary or nullary.

Corollary 6. Let $L^{*}$ be any expansion of a logic $L$ with new compatible connectives and INT $\subseteq L$. If $\neg \neg A \vee \neg A \in L$, then unification in $L^{*}$ is filtering, hence it is unitary or nullary.

Corollary 7. Let $L^{*}$ be an expansion of a logic $L$ with new implicit compatible operations from $\{S, \gamma, G\}$ and $I N T \subseteq L$. If $\neg \neg A \vee \neg A \in L$, then unification in $L^{*}$ is filtering, and hence it is unitary or nullary.

Remarks. Adding a new compatible operation $f$, satisfying additional axioms (which are regarded as an implicit definition of $f$ ), to some variety $\mathcal{V}$ of Heyting algebras usually produces a new, variety $(\mathcal{V}, f)$ which does not contain some algebras from $\mathcal{V}$. Also $(\mathcal{V}, f)$ has different properties than $\mathcal{V}$, especially different are subalgebras, free algebras (for instance, 1generated free algebra of 3-valued Gödel logic has 6 elements, but with the successor added it has 15 elements, see 6.1 of [4]). Also projective algebras are different. Since Ghilardi proved [12] that unification depends on projective objects there is no reason to expect that the unification types are preserved after adding compatible operations.

Hence, naturally, some compatible operations are not defined on every Heyting algebra. For instance, $S$ can not be defined on the unit interval of the real numbers $[0,1]$ (see 5.2 of [4]). This fact is used for proving that the operation $f$ is new: assume, to the contrary, that $f$ is not new, so it can be defined, i.e. $f(x)=t(x)$, for some Heyting term $t$. Then $f(x)=t(x)$ would exist on every algebra of $\mathcal{V}$. Compatible operations have connection with affine completeness. An algebra $\mathrm{H}$ is affine complete if any compatible function of $\mathrm{H}$ is given by a polynomial of $\mathrm{H}$ (K. Kaarli, A. Pixley 2000). For example, Boolean algebras (Grätzer), finite Heyting algebras are affine complete, but infinite Heyting algebras, in general, are not.

Unifiability and consistency. It is well known that in every intermediate logic unifiable formulas and consistent formulas coincide. We claim that adding compatible operations (connectives) makes significant changes 
in unification. To support this claim we provide (in Heyting algebras with additional compatible connectives) terms (formulas) which are satisfiable (or consistent) but not unifiable. Let $\mathcal{B}$ be the variety of Boolean algebras.

LEMma 8. Let $\tau$ be a frontal operator on a variety $\mathcal{V}$ of Heyting algebras, $\mathcal{V} \neq \mathcal{B}$, such that $1 \neq \tau(0) \neq 0$. Then the term $x \wedge \tau(\neg x)$ is satisfiable (or consistent) but $(x \wedge \tau(\neg x), 1)$ is not unifiable in $\mathcal{V}$.

Proof: Let $\mathcal{V}$ be a variety of Heyting algebras. Assume that $x \wedge \tau(\neg x)$ is unifiable in $\mathcal{V}$, i.e. for some substitution $\sigma$ we have $1=\sigma(x \wedge \tau(\neg x))=$ $\sigma(x) \wedge \tau(\neg \sigma(x))$. Hence $\sigma(x)=1$ and $\tau(\neg \sigma(x))=1$, but $\tau(\neg \sigma(x))=$ $\tau(\neg 1)=\tau(0) \neq 1$, a contradiction.

On the other hand, since $\mathcal{V} \neq \mathcal{B}$ we have $\mathcal{C}_{3} \subseteq \mathcal{V}$, where $\mathcal{C}_{3}$ is a variety of Heyting algebras generated by a 3 -element chain $C_{3}$. Thus $C_{3} \in \mathcal{V}$ and let $a \in C_{3}$ be such that $0 \neq a \neq 1$. Now, for a valuation $v$ such that $v(x)=a$ we have $v(x \wedge \tau(\neg x))=a \wedge \tau(0)=a \neq 0$, since $\tau(0) \neq 0$. Hence $x \wedge \tau(\neg x)$ is satisfiable (consistent) in $\mathcal{V}$.

Note that if $\mathcal{A} \notin \mathcal{B}$ then: $0 \neq S(0) \neq 1$ and $0 \neq \gamma(0) \neq 1$ in $\mathcal{A}$.

Corollary 9. For every variety of Heyting algebras $\mathcal{V} \neq \mathcal{B}$ with a frontal operation $\tau$ such that $0 \neq \tau(0) \neq 1$, there are terms which are satisfiable (consistent) but not unifiable. In particular, $x \wedge S(\neg x)$ and $x \wedge \gamma(\neg x))$ are such.

Residuated lattices. Now we consider analogous results in expansions of bounded integral commutative residuated lattices with compatible operations, where the Stone identity $\neg \neg x \vee \neg x=1$ holds in the lattices. An algebra $\mathcal{L}=(L, \wedge, \vee, \cdot, \rightarrow, 0,1)$ is called a bounded integral commutative residuated lattice, or simply bounded residuated lattice, if

(1) $(L, \wedge, \vee, 0,1)$ is a bounded lattice;

(2) $(L, \cdot)$ is a commutative monoid with unit element 1 ;

(3) $x \cdot y \leq z \Leftrightarrow x \leq y \rightarrow z$, for all $x, y, z^{\prime}$

and moreover, we define $\neg x=x \rightarrow 0$.

For more information on residuated lattices and for references see [11] and [16]. The variety of bounded residuated lattices is 1-regular. It is shown in [9] that any bounded residuated lattice is an $l$-algebra satisfying condition $(\mathrm{A})$. 
THEOREM 10 ([9]). Unification in commutative integral residuated lattices satisfying the Stone identity $\neg \neg x \vee \neg x=1$ is filtering, that is, unitary or nullary.

COROLLARY 11. Unification in commutative integral residuated lattices satisfying the Stone identity $\neg \neg x \vee \neg x=1$ with additional compatible operations is filtering, that is, unitary or nullary.

In commutative residuated lattices a family of compatible operations $S_{n}, n \geq 1$, is considered, see [5], such that $S_{1}$ corresponds to the operation $S$ in Heyting algebras. If some conditions hold (see [5]) in the residuated lattice, then $S_{n}$, defined by the formula: $S_{n}(x)=\min \left\{y: y^{n} \rightarrow x \leq y\right\}$, is compatible (it may happen that $S_{1}$ is defined but $S_{2}$ is not, etc.). Not much is known about unification types in commutative integral bounded residuated lattices with additional compatible operations, e.g. with $S_{n}$.

COROLlARY 12. Unification in commutative integral residuated lattices satisfying the Stone identity $\neg \neg x \vee \neg x=1$ with additional compatible operations $S_{n}$, if defined, is filtering, that is, unitary or nullary.

There are many questions in the area presented above, that have not been answered yet.

\section{References}

[1] F. Baader, S. Ghilardi, Unification in Modal and Description Logics, Logic Journal of the IGPL, vol. 19 (6), (2011), pp. 705-730.

[2] F. Baader, W. Snyder, Unification Theory. in A. Robinson, A. Voronkov, (eds.) Handbook of Automated Reasoning, Elsevier Science Publisher, (2001).

[3] S. Burris, H. P. Sankappanavar, A Course in Universal Algebra, The Millennium Edition, Burris, S., Sankappanavar, H. P.

[4] X. Caicedo, R. Cignoli, Algebraic Approach to Intuitionistic Connectives Journal of Symbolic Logic 66 (2001), pp. 1620-1636.

[5] J. L. Castiglioni, M. Menni and M. Sagastume, Compatible operations on commutative residuated lattices, Journal of Applied Non-Classical Logics 18 (2008), pp. 413-425.

[6] J. L. Castiglioni, H. J. San Martn, Compatible Operations on Residuated Lattices, Studia Logica 98 (2011), pp. 203-222. 
[7] P. Cintula, G. Metcalfe, Admissible rules in the implicationnegation fragment of intuitionistic logic, Annals of Pure and Applied Logic 162, 2 (2010), pp. $162-171$.

[8] W. Dzik, Splittings of Lattices of Theories and Unification Types, Contributions to General Algebra 17 (2006), pp. 71-81.

[9] W. Dzik, S. Radeleczki, Direct Product of l-algebras and Unification. An Application to Residuated Lattices, Journal of Multiple-valued Logic and Soft Computing 28(2-3) (2017), pp. 189-215.

[10] L. Esakia, The modalized Heyting calculus: a conservative modal extension of the Intuitionistic Logic, Journal of Applied Non-Classical Logics 16, No. 3-4 (2006), pp. 349-366.

[11] N. Galatos, P. Jipsen, T. Kowalski, H. Ono, Residuated Lattices. An Algebraic Glimpse at Substructural Logics, Studies in logic and the foundations of mathematics, Vol 151, Amsterdam: Elsevier, 2007.

[12] S. Ghilardi, Unification through Projectivity, Journal of Symbolic Computation 7 (1997), pp. 733-752.

[13] S. Ghilardi, Unification in Intuitionistic Logic, Journal of Symbolic Logic 64(2)(1999), pp. 859-880.

[14] S. Ghilardi, L. Sacchetti, Filtering Unification and Most General Unifiers in Modal Logic, Journal of Symbolic Logic 69 (2004), pp. 879-906.

[15] S. Ghilardi, Best Solving Modal Equations, Annals of Pure and Applied Logic 102 (2000), pp. 183-198.

[16] T. Kowalski, H. Ono, Residuated lattices: An algebraic glimpse at logics without contraction, (2001), manuscript.

[17] A. V. Kuznetsov, On intuitionistic propositional provability calculus, Soviet Math. Doklady 32 (1985), pp. 27-30.

[18] A. Wronski, On factoring by compact congruences in algebras of certain verieties related to the intuitionistic logic, Bulletin of the Section of Logic 28 (1986), pp. 48-50.

University of Silesia

Institute of Mathematics, Bankowa 12

40007 Katowice, Poland

e-mail: wojciech.dzik@us.edu.pl
University of Miskolc, Institute of Mathematics 3515 Miskolc-Egyetemváros, Hungary e-mail:matradi@uni-miskolc.hu 